\title{
Benefit Corporation : Faut-il introduire en France une nouvelle forme d'entreprise lucrative ayant l'obligation d'être utile socialement ou environnementalement ?
}

\author{
Gurvan Branellec \\ Docteur en droit, Enseignant-chercheur en droit, \\ Brest Business School, \\ Responsable du Programme Bachelor Campus de Brest. \\ Chercheur associé UMR AMURE. \\ gurvan.branellec@france-bs.com
}

\author{
Ji-Yong Lee \\ Docteur en Sciences Economiques, Enseignant-chercheur en finance, \\ Brest Business School, \\ ji-yong.lee@france-bs.com
}

La Benefit Corporation est donc une forme juridique hybride qui tient des sociétés capitalistes et des organisations à but non-lucratif (1.1). Grâce à cette forme juridique il devient possible de réconcilier les intérêts des actionnaires avec ceux des parties-prenantes formant l'entreprise et d'ancrer dans les fondements mêmes de l'entreprise le principe de responsabilité sociale (1.2). Si en théorie cette nouvelle forme juridique semble ouvrir la voie vers des entreprises plus responsables, la mise en pratique semble plus complexe en ce qui concerne notamment l'impératif de concilier une finalité sociale et environnementale et la maximisation des profits (2.1). Cette forme juridique présente toutefois l'avantage de permettre à une entreprise de formaliser son engagement en matière sociale et environnementale. Dès lors la question de la pertinence de leur introduction en France se pose (2.2).

Mots-clés : Benefit Corporation, responsabilité sociale des entreprises, économie sociale et solidaire, gouvernance d'entreprise, entreprise à but lucratif

\section{Benefit Corporation: Should France introduce a new for-profit business models driven by socially or environmentally mission?}

The Benefit Corporation is a hybrid legal form because it attempts to blend aspects of for-profit organizations and not-for-proft 
organizations (1.1). This legal form permits corporate directors to consider stakeholders' interests as well as the financial interests of shareholders by implementing corporate social responsibility (1.2). Theoretically speaking if this new legal form enables organizations to operate in a socially and environmentally responsible manner, it can also be expected to have substantial difficulty easing the friction between shareholder wealth maximization and social and environmental purposes (2.1). However, this legal form helps foster the capacity and internal motivation necessary for corporations to engage in a socially and environmentally responsible manner. Therefore the relevance of its introduction in France is questioned (2.2).

Key-words: Benefit Corporation, corporate social responsibility, Social and solidarity-based economy, corporate governance, for-profit company

\section{Benefit Corporation: Debe Francia introducir una nueva forma de empresa lucrativa con obligación de ser socialmente o ambientalmente útil?}

La Benefit Corporation es una forma jurídica híbrida entre las empresas capitalistas y las organizaciones de objetivo sin lucro (1.1). Gracias a esta forma jurídica es posible conciliar los intereses de los accionistas y los de las partes interesadas que constituyen la empresa. También es posible inscribir en los fundamentos de la empresa el principio de responsabilidad social (1.2). En teoría, con esta nueva forma jurídica es posible tener empresas más responsables. Pero, la realidad, parece más compleja por lo que es de la obligación de conjugar una finalidad social y medioambiental con la maximización del lucro (2.1). Esta forma jurídica tiene la ventaja de permitir que una empresa pudiera formalizar su implicación a nivel social y medioambiental. Entonces, nos podemos preguntar sobre la pertinencia de introducir este modelo jurídico en Francia (2.2).

Palabras claves: Benefit Corporation, responsabilidad social de la empresa, economia social y solidaria, Gobernancia de la empresa, empresa con finalidad lucrativa 


\section{Introduction}

Les dernières décennies ont été marquées par un vaste processus de transformations économiques et politiques à l'échelle de la planète. La finance constitue une grande force à l'origine de ces mouvements. La montée en puissance de la finance de marché va de pair avec de profondes mutations des formes de gouvernance des entreprises : elle subordonne l'organisation de la firme aux exigences de la maximisation de la rentabilité financière. En d'autres termes, la « création de valeur » pour l'actionnaire est devenue l'objectif de la gouvernance dans les grandes entreprises. Or, le comportement d'une entreprise n'est pas uniquement susceptible d'affecter les créateurs ou dirigeants, il peut également concerner d'autres parties prenantes internes (adhérents, salariés...) ou externes (consommateurs, créanciers, clients, citoyens...). La crise financière actuelle met en cause ce modèle de valeur actionnariale. Nous sommes progressivement passés à une approche élargie qui tient compte d'autres parties prenantes telles que les salariés, les fournisseurs, la collectivité et l'environnement. La gouvernance doit guider et réguler la construction d'une entreprise durable et responsable à l'égard des parties prenantes et de l'environnement (Martinet, 2008, p.105). L'entreprise est en effet un lieu ouvert où se rencontrent différents types de parties prenantes qui interagissent entre elles (Aglietta et Rebérioux, 2004, p. 349; Blair, 1995, p. 17; Charreaux, 1997, p. 422).

Le concept de responsabilité sociale de l'entreprise (RSE) permet de faire entrer les parties prenantes dans la gouvernance d'entreprise grâce aux valeurs sociales, environnementales et éthiques. Cette prise en compte par les entreprises d'une responsabilité sociétale est assez ancienne (mouvement coopératif ou mutualiste, mécénat, politique sociale de grandes entreprises) mais elle se développe aujourd'hui à cause des pressions sociales, environnementales et économiques (Desbarats, 2008, p. 68). La société civile amène les entreprises à adopter des démarches de responsabilité sociale. Il peut s'agir d'une démarche sincère d'entreprises soucieuses de considérations éthiques, ou d'un souhait d'éviter des mises en jeu de leur responsabilité voire encore d'une stratégie marketing visant à améliorer leur image de marque (une entreprise capitaliste peut souhaiter améliorer son image de marque en créant une filiale qui serait une entreprise sociale et solidaire). Cette prise en compte par les entreprises de leur responsabilité sociale se caractérise surtout par des démarches volontaires. 
Les entreprises se dotent de chartes sociales, codes de conduite, codes de déontologie, référentiels d'engagement... Il s'agit de «signes de qualités privés » (Blin-Franchomme, 2009, p. 72) qui se développent en dehors de la contrainte de la loi. La Commission européenne met l'accent sur cette autorégulation des entreprises en définissant la RSE comme «l'intégration volontaire des préoccupations sociales et écologiques des entreprises à leurs activités commerciales et leurs relations avec les parties prenantes $»^{1}$ et a précisé en 2011 que «la responsabilité sociale des entreprises concerne les actions de celles-ci qui vont au-delà des obligations juridiques qui leur incombent à l'égard de la société et de l'environnement $»^{2}$.

Ces initiatives privées, constitutives d'un droit souple (soft law), se rencontrent dans des domaines variés: accords environnementaux (éco-labels, réduction des emballages...), engagements sociaux (développement durable, commerce équitable...), règles de conduite financière ou éthique (rémunération des dirigeants, non-discrimination, amélioration des conditions de travail des salariés)... Les domaines concernés, ainsi que les valeurs consacrées, sont d'une grande diversité. La forme de ces engagements et les formules employées le sont tout autant. Ces démarches volontaires peuvent aller de formules floues qui comportent des engagements imprécis jusqu'à des adjonctions aux règlements internes des entreprises (Pereira, 2009, p. 30) ou des engagements pris par des entreprises ou branches sectorielles qui vont produire des conséquences juridiques. Ces chartes et codes de conduite permettent aux entreprises de formaliser et de communiquer sur une démarche responsable.

Ainsi, il apparaît que toutes les entreprises doivent prendre en compte leurs impacts sociaux, sociétaux et environnementaux. La mise en œuvre d'une démarche RSE est devenue une nécessité, un atout stratégique inévitable pour les entreprises quelle que soit leur taille ou leur activité. La prise en compte des parties prenantes, passe nécessairement par une intégration de celles-ci et de leurs intérêts.

Aux États-Unis, la volonté d'intégrer le modèle de responsabilité de l'organisation au travers de ses relations avec les parties prenantes donne naissance à une nouvelle forme d'entreprise dénommée «Benefit Corporation». Il s'agit d'un nouveau statut

\footnotetext{
${ }^{1}$ Livre vert, juillet 2001.

${ }^{2}$ Responsabilité sociale des entreprises: une nouvelle stratégie de l'UE pour la période 2011-2014, Communication de la Commission au Parlement européen, au Conseil, au Comité économique et social européen et au Comité des régions. Com (2011), 681.
} 
juridique pour les entreprises qui ont pour mission de créer un impact social et environnemental positif. La Benefit Corporation se veut donc le porte-drapeau d'un nouveau type d'entreprises ayant une utilité sociale et environnementale (1). Ce nouveau modèle conçu comme un réel instrument de RSE méritait d'être éclairé pour examiner sa possible transposition en France (2).

\section{La Benefit Corporation : la naissance d'une entreprise sociale et environnementale}

Le modèle de gouvernance actionnariale laisse place à un nouveau modèle d'entreprises sous l'impulsion de préoccupations sociales et environnementales. Il s'agit de la Benefit Corporation : un statut qui permet aux entreprises d'inclure des intérêts extra-financiers tout en recherchant le profit. La création de ces Benefit Corporations à fait évoluer récemment le droit traditionnel des sociétés dans plusieurs États américains (1.1) en créant, entre les organisations à but lucratif et celles à but non-lucratif une catégorie hybride qui intègre à la fois des préoccupations sociales, environnementales et économiques (1.2).

\subsection{Un hybride entre organisations à but lucratif et organisation à but non-lucratif}

Inventées aux États-Unis en 2010, les Benefit Corporations sont une nouvelle forme d'entreprises qui vise à satisfaire des objectifs sociétaux ou environnementaux sans pour autant renoncer aux profits ${ }^{3}$. Il s'agit d'une entité juridique hybride, à mi-chemin entre une entreprise classique et une association sans but lucratif (Resor, 2012,

\footnotetext{
${ }^{3}$ Il ne faut pas confondre le concept de Benefit Corporation avec celui de Certified B Corporation. Souvent les deux sont appelés $B$ Corps par erreur ou comme une abréviation. La Certified B Corporation est une société qui a obtenu la certification permettant de souligner l'engagement durable et responsable d'une entreprise pour répondre à des enjeux sociaux ou environnementaux. Il s'agit du label $B$ Corporation . Cette certification est délivrée par un organisme sans but lucratif, B Lab, créé en 2007 par Bart Houlahan, Jay Coen Gilbert et Andrew Kassoy. Aujourd'hui, 707 entreprises sont certifiées B Corporations opérant dans 60 secteurs d'activité différents dans 24 pays. A la différence d'une entreprise certifiée B Corporation, la Benefit Corporation est une forme juridique en vigeur dans un État. Pour obtenir le label B Corporation, l'entreprise doit respecter au moins 80 critères sur 200 points du questionnaire $B$ Impact Assessment (sur le plan des employés, des consommateurs, de l'environnement ou encore la transparence des comptes). Par contre, la Benefit Corporation doit publier des informations sociales et environnementales dans le rapport annuel de gestion mais ce rapport n'est pas tenu d'être vérifié, certifié, ou audité par un organisme tiers indépendant.
} 
p. 101). D'un côté, les gouvernements et associations sans but lucratif n'arrivent pas à répondre à tous les problèmes de la société d'aujourd'hui. D'un autre côté, les dirigeants d'entreprises capitalistes qui souhaitent engager leur entreprise dans une démarche sociale et/ou environnementale prennent le risque d'une mise en jeu de leur responsabilité, même par des actionnaires minoritaires, si cette démarche ne va pas dans le sens d'une maximisation des profits. En effet, la règlementation américaine ne protège les dirigeants que d'une mise en jeu de leur responsabilité personnelle (Business Judgement Rule) tant que leurs décisions sont prises de bonne foi, dans l'intérêt de la société, de façon prudente et informée. Elle ne les protège toutefois pas lorsque leurs décisions de gestion profitent explicitement à d'autres parties prenantes que les seuls actionnaires (Levillain, 2012, $\mathrm{n}^{\circ} 450$ ).

De là est née l'idée de la Benefit Corporation, une création d'une nouvelle forme juridique qui entend utiliser la force des entreprises pour créer un bénéfice public. Cette nouvelle structure légale progresse aux États-Unis : depuis 2010, vingt États américains ont adopté des lois permettant aux sociétés de revendiquer leurs statuts d'entreprises responsables : le Maryland, le Vermont, la Virginie, le New Jersey, Hawaii, la Californie, New York, la Pennsylvanie, la Louisiane, l'Illinois, la Caroline du Sud, le Massachusetts, le Colorado, l'Arizona, le Nevada, Washington DC, Rhode Island, l'Oregon, le Delaware et l'Arkansas ${ }^{4}$.

\footnotetext{
4 Voir site de la Benefit Corporation, http://www.benefitcorp.net/state-by-statelegislative-status.

La législation de la Californie a été promulguée le 9 Octobre 2011 et est entrée en vigueur le 1er Janvier 2012. La législation d'Hawaii a été promulguée et est entrée en vigueur le 8 Juillet 2011. La législation de l'Illinois a été promulguée le 2 Août 2012 et est entrée en vigueur le 1er Janvier 2013. La législation de la Louisiane a été promulguée le 31 mai 2012 et est entrée en vigueur le 1er Août 2012. La législation du Maryland a été promulguée le 13 Avril 2010 et est entrée en vigueur le 1er Octobre 2010. La législation du Massachusetts a été promulguée le 7 Août 2012 et est entrée en vigueur le 1er Décembre 2012. La législation de New Jersey a été adoptée le 10 Janvier 2011, a été promulguée et est entrée en vigueur le 1er Mars 2011. La législation de New York a été promulguée le 12 Décembre 2011 et est entrée en vigueur le 10 Février 2012. La législation de Pennsylvanie a été promulguée le 24 Octobre 2012 et est entrée en vigueur le 22 Janvier 2013. La législation de la Caroline du Sud a été promulguée et est entrée en vigueur le 14 Juin 2012. La législation du Vermont a été promulguée le 19 Mai 2010 et est entrée en vigueur le 1er Juillet 2011. La législation de la Virginie a été promulguée le 26 Mars 2011 et est entrée en vigueur le 1er Juillet 2011. La législation du Colorado a été promulguée le 15 Mai 2013 et entrera en vigueur le 1er Avril 2014. La législation de l'Arizona a été promulguée le 30 Avril 2013 et entrera en vigueur le 31 Décembre 2014. La législation du Nevada a été promulguée le 24 Mai 2013 et est entrée en vigueur le 1er Janvier 2014. La législation de Washington DC a été promulguée le 28 Janvier 2013 et est entrée en vigueur le 1er Mai 2013. La législation
} 
Les Benefit Corporations sont tenues d'avoir une mission précise afin de créer un «bénéfice public général» et elles s'engagent à identifier certains objectifs spécifiques non lucratifs, dits «bénéfices publics spécifiques». Cela diffère des entreprises n'utilisant pas ce type de forme juridique, qui doivent uniquement avoir un objet social licite. Le «bénéfice public general » est défini comme "a material, positive impact on society and the environment, as measured by a third party standard, through activities that promote a combination of specific public benefits" (State of Maryland, 2010, p.2) ${ }^{5}$. La fonction des Benefit Corporations est presque identique dans les lois de chaque État américain ${ }^{6}$. Ces différentes lois ont mis en place le même modèle de législation ${ }^{7}$ qui énumère sept possibilités de «bénéfices publics spécifiques» qui sont:

- Fournir aux personnes à faibles revenus ou mal desservies ou aux communautés, des produits ou services ;

- Promouvoir les possibilités économiques pour les individus ou les communautés au-delà de la création d'emplois ;

- Préserver l'environnement ;

- Améliorer la santé humaine ;

- Promouvoir les arts, les sciences, ou l'avancement des connaissances ;

- Fournir des capitaux à des entités avec un but d'utilité publique ;

- L'accomplissement de toute autre prestation particulière pour la société ou l'environnement.

Certains États, et notamment la Caroline du Sud, ont ajouté la possibilité d'un but religieux. La poursuite de ce but religieux ne

de Rhode Island a été promulguée le 17 Juillet 2013 et est entrée en vigueur le 1er Janvier 2014. La législation de l'Oregon a été promulguée le 04 Juin 2013 et est entrée en vigueur le 1er Janvier 2014. La législation du Delaware a été promulguée le 17 Juillet 2013 et est entrée en vigueur le 1er Août 2013. La législation de l'Arkansas a été promulguée le 19 Mai 2013 et est entrée en vigueur le 19 Septembre 2013. D'autres États envisagent également d'adopter une telle législation : l'Alabama, le Connecticut, le Kentucky, l'Utah ou encore le Minnesota.

${ }^{5}$ Le premier État américain à avoir intégré dans sa loi le statut de Benefit Corporation en avril 2010 .

${ }^{6}$ Supra note de bas de page $\mathrm{n}^{\circ} 2$.

${ }^{7}$ Il s'agit d'un modèle-type permettant de préparer la rédaction de législation applicable à la Benefit Corporation, sous réserve que le modèle soit modifié selon les États. Ce modèle de législation a été rédigé par Bill Clark de Drinker, Biddle, \& Reath LLP (cabinet d'avocats). Il évolue en fonction des commentaires des avocats d'affaires des États qui ont adopté la Benefit Corporation. Modèle-type permettant de préparer la rédaction de législation applicable à la Benefit Corporation, sous réserve que le modèle soit modifié selon les États. 
dispense toutefois pas la Benefit Corporation de générer un «bénéfice public général» (Esposito et Pelsinger, 2014). Les Benefit Corporations ont en fait pour particularité d'intégrer des objectifs de RSE en poursuivant la recherche d'un «bénéfice public général». Une des spécificités de la Benefit Corporation repose sur l'idée que les objectifs sociétaux ou environnementaux ne doivent pas nécessairement primer sur la recherche du profit à la différence d'une organisation non-lucrative (Figure 1). Il convient dès lors de ne pas confondre la Benefit Corporation avec d'autres structures alternatives adaptées aux enjeux de la RSE créées aux États-Unis : la Low Profit Limited Liability Company (L3C) et la Flexibility Purpose Corporation (FPC).

Figure 1 : L'émergence de nouveau modèle d'entreprise

\begin{tabular}{|c|c|c|c|}
\hline Secteur public & Secteur social & Nouveau secteur & Secteur privé \\
\hline $\begin{array}{c}\text { Entreprise } \\
\text { Gouvernementale }\end{array}$ & $\begin{array}{l}\text { Entreprise } \\
\text { sans } \\
\text { but lucratif }\end{array}$ & $\begin{array}{c}\text { Entreprise } \\
\text { Hybride } \\
\\
\text { Benefit } \\
\text { Corporation } \\
\\
\text { Les objectifs } \\
\text { sociétaux ou } \\
\text { environnementaux } \\
+ \\
\text { La recherche du } \\
\text { profit }\end{array}$ & $\begin{array}{l}\text { Entreprise } \\
\text { à } \\
\text { but lucratif }\end{array}$ \\
\hline
\end{tabular}

Source : figure modifiée d'André (2012, p. 135).

La L3C est proche de la Limited Liability Company traditionnelle mais ce type de société a davantage de latitude pour mener une politique d'entreprise en matière sociale et environnementale. Contrairement à la Benefit Corporation qui n'abandonne pas la recherche du profit, la L3C reconnaît expressément que sa mission sociale est prioritaire sur son objectif de profitabilité. En revanche, la FPC poursuit juste un objectif particulier qui pourrait avoir un effet positif sur ses parties prenantes (notamment ses employés, fournisseurs, clients, créanciers, de la société, ou de l'environnement). Elle n'est pas contrainte d'avoir une vérification des objectifs sociétaux ou environnementaux par un tiers comme dans la 
Benefit Corporation. Cette société se distingue également de la Community Interest Company (CIC) créé en 2005 au Royaume-Uni en ce qu'il n'y a pas de règlementation de son objet social ou de son activité et que les droits aux profits des actionnaires ne sont pas limités.

Pour obtenir le statut d'une Benefit Corporation, l'entreprise doit remplir trois conditions :

- Créer un «bénéfice public général» définit comme un impact positif sur la société et l'environnement. Cet objectif permet de s'assurer que le «bénéfice public général» l'emporte sur les intérêts financiers. La prise de décision au sein de ces sociétés n'est plus uniquement liée aux enjeux financiers.

- Prendre en compte l'impact des décisions prises par les organes de gouvernance de la société sur les parties prenantes.

- Publier un rapport annuel sur ses performances sociales et environnementales.

La création d'une Benefit Corporation est un acte volontaire. Cette forme juridique peut aussi bien être choisie par des créateurs d'entreprise que par des dirigeants d'une entreprise existante. Pour créer une nouvelle entreprise sous cette forme, il suffit d'opter pour le statut de Benefit Corporation dans un État où cette nouvelle forme juridique d'entreprise existe. Si l'entreprise existe déjà sous une autre forme juridique il faudra une décision de l'assemblée des actionnaires statuant à une majorité des deux tiers ${ }^{8}$. De même, lors d'une fusion ou d'une acquisition, la réunion d'au moins deux tiers des voix des actionnaires est nécessaire si l'entité absorbante n'a pas de gouvernance de type Benefit Corporation.

Cette nouvelle forme juridique d'entreprises fait entrer le droit des sociétés ${ }^{9}$ américain dans une nouvelle ère de mutations. Jusqu'à présent, les différentes règlementations concernant le droit des sociétés aux États-Unis prévoyaient que l'entreprise devait chercher à maximiser les profits pour les actionnaires ${ }^{10}$. La seule obligation des

\footnotetext{
${ }^{8}$ Model Benefit Corporation Legislation.

${ }^{9}$ Il s'agit des règles juridiques qui définissent les relations entre les différents constituants de l'entreprise, soit les actionnaires, les administrateurs, les dirigeants et les salariés. Aux États-Unis, le droit des sociétés n'est pas fixé par une loi fédérale mais dépend de la compétence de chaque État.

10 "A director or officer has a duty to the corporation to perform the director's or officer's functions...in a manner that he or she reasonably believes to be in the best interests of the corporation....." (American Law Institute, 1994, p. section 4.01)

"Thus the corporation should have as its objective the conduct of business activities with a view to enhancing corporate profit and shareholder gain", Ibid., section 2.01 .

Bien que le droit des sociétés soit spécifique à chaque législation en vigueur dans l'État, le concept général des obligations fiduciaires est standard dans presque toutes les
} 
dirigeants d'entreprises envers leurs actionnaires était donc la recherche du profit. Historiquement, le système juridique américain régissant les personnes morales et leurs activités n'a pas été élaboré pour permettre à des entreprises à but lucratif de développer une action en matière environnementale et sociale. En effet, la société appartenant aux actionnaires et ces derniers ayant le pouvoir de remercier le directeur général, il n'a à rendre de comptes qu'à eux.

La règlementation américaine rendait ainsi difficile la prise en compte par les dirigeants des enjeux sociaux et environnementaux dans leurs processus décisionnels. La Benefit corporation, nouveau type d'entreprise rendant impératif cette prise en compte, permet aux entreprises d'intégrer davantage leurs parties prenantes dans leur gouvernance ${ }^{11}$. Même s'il n'y a pas encore de jurisprudence spécifique aux Benefit Corporations, la Cour Suprême des États Unis ${ }^{12}$ a reconnu cette forme juridique hybride à l'occasion d'une décision autorisant les for-profit corporations à poursuivre un but financier et des fins religieuses. La Cour Suprême souligne à cette occasion que la Benefit Corporation est une entité qui a à la fois pour objectif de fournir des profits à ses propriétaires et un bénéfice pour le public.

\subsection{Une société socialement et environnementalement responsable dans son ADN}

Pourquoi manifester un intérêt particulier pour ce statut ? Cette nouvelle voie législative permet de faire la distinction entre des entreprises soucieuses de leur environnement uniquement à des fins marketings ou commerciales et des entreprises soucieuses de bâtir un monde économique plus durable.

Les Benefit Corporations doivent répondre à des standards sociaux et environnementaux et attester de leur respect dans le «rapport annuel des bénéfices » (à destination du public et des

juridictions américaines. Les devoirs fiduciaires par la direction constituent une clef importante du droit des sociétés aux États-Unis.

"even if corporate profit and shareholder gain are not thereby enhanced, the corporation, in the conduct of its business: ... may take into account ethical considerations that are reasonably regarded as appropriate to the responsible conduct of business; and may devote a reasonable amount of resources to public welfare, humanitarian, educational, and philanthropic purposes.", Ibid., section 2.01.

${ }^{11}$ Aujourd'hui 1082 entreprises dans vingt États américains ont adopté le statut de Benefit Corporation. Voir le site de la Benefit Corporation, http://benefitcorp.net/finda-benefit-corp/search.

${ }^{12}$ Supreme Court of the United States, 573 U.S., Burwell v. Hobby Lobby Stores, Inc., $30 / 06 / 2014$ 
investisseurs). L'entreprise qui adhère au principe de gouvernance «Benefit corporation» doit ainsi justifier qu'elle poursuit bien un bénéfice public. Elle doit également y faire mention du périmètre d'action et des mesures concrètement mises en place pour créer de la valeur dans une logique socialement et écologiquement responsable ${ }^{13}$. Ce rapport doit être évalué par un organisme tiers indépendant. Le recours à un tiers indépendant est un élément important de la Benefit Corporation car celui-ci va permettre à une entité de se rattacher au statut des Benefit Corporations et il va ensuite jouer un rôle d'évaluation de la performance sociale et environnementale des entreprises ${ }^{14}$. Ces dispositions ont pour but de fournir aux actionnaires, parties prenantes, et même aux investisseurs extérieurs des informations exactes et claires, sur le respect par la société de ses objectifs (Esposito, 2013, p. 660). Le choix de l'organisme tiers indépendant est fait par la Benefit Corporation qui peut avoir recours à des organismes du type : B Lab, Global Reporting Initiative (GRI), GreenSeal, Underwriters Laboratories (UL), Green America. De nombreux organismes chargés de l'élaboration des normes et certifications relative à la responsabilité sociale des entreprises peuvent également certifier le statut d'une Benefit Corporation.

Une autre caractéristique importante des Benefit Corporations est l'obligation des dirigeants de considérer les intérêts des différentes parties prenantes (essentiellement employés, clients, fournisseurs, communautés et environnement) ainsi que de tout autre facteur pertinent lorsqu'ils prennent des décisions dans le meilleur intérêt de l'entreprise ${ }^{15}$. La prise en compte de l'ensemble des partenaires de l'entreprise distingue la Benefit Corporation de la société classique dont l'objectif unique est de maximiser son profit au bénéfice de ses actionnaires (Munch, 2012, p. 171). La théorie des parties prenantes, qui s'intéresse à un ensemble plus vaste d'acteurs, étant un cadre théorique dominant pour la RSE, la Benefit Corporation apparait alors comme un levier justifiant la poursuite de la responsabilité sociale des entreprises ${ }^{16}$. Ainsi, ce nouveau type d'entreprise permet de protéger les dirigeants qui ne souhaitent pas que leur entreprise soit uniquement

\footnotetext{
${ }^{13}$ State of Maryland, Maryland General Assembly (2010).

${ }^{14}$ Maryland Code corporations and associations, 5-6C-08.

"Third-party standard" is defined as "a recognized standard for defining, reporting and assessing overall corporate social and environmental performance".

${ }^{15}$ Supra note de bas de page 11, 5-6C-07.

${ }^{16}$ Le souci d'élargir la réflexion à la question de la responsabilité des entreprises à l'égard des parties prenantes autres que les actionnaires a conduit à proposer des approches partenariales (Charreaux et Desbrières, 1998, p. 58; Tirole, 200, p. 5; Zingales, 1997, p. 13).
} 
mise au service des intérêts de ses actionnaires. Les dirigeants ont un rôle d'arbitrage entre les diverses parties prenantes, c'est-à-dire entre les exigences de l'institution financière et de l'entreprise réelle (Brechet et Tougeron, 2009, p. 22 ; Hafsi et Youssofzai, 2008, p. 128). Les dirigeants responsables sont déchargés de toute responsabilité en cas de dommages financiers tant que leurs décisions ont été prises de bonne foi dans l'intérêt général et de façon prudente (afin de ne pas inquiéter les dirigeants quant aux engagements qu'ils ont pris volontairement).

De même, ils sont protégés face à d'éventuels procès qui pourraient émaner des parties prenantes, lesquelles n'ont pas de droits de poursuites judiciaires ${ }^{17}$. Les actionnaires demeurent toutefois des parties prenantes importantes à considérer dans la Benefit Corporation car cette nouvelle forme de gouvernance ne se dégage pas totalement du contrôle des actionnaires ${ }^{18}$. Une des missions des dirigeants est donc de concilier les intérêts des partenaires financiers avec ceux des autres parties-prenantes qui font partie de l'entreprise.

En fin de compte, les éléments mentionnés ci-dessus nous permettent de mieux cerner les divers intérêts qu'apporte la Benefit Corporation : légitimation de la mission sociétale, protection légale face aux décisions non-financières, droit d'accomplir un autre objectif que la maximisation des profits, règles de transparence et de responsabilité, etc. (Resor, 2012, p. 105). Un autre intérêt de l'introduction de cette nouvelle entreprise hybride est aussi d'attirer dans son capital un nouveau type d'investissement : l'investissement socialement responsable. Grâce à son respect des critères en termes de performance sociale et environnementale ainsi que de transparence, la Benefit Corporation pourrait plaire aux investisseurs qui ne s'intéressent plus uniquement aux caractéristiques financiers mais également aux performances extra-financières.

\section{Une nouvelle forme juridique dont l'utilité est encore controversée}

La Benefit Corporation se veut un moyen de permettre à des entreprises de concilier une finalité économique et une utilité sociale et environnementale. Leur mise en œuvre aux États-Unis a toutefois

\footnotetext{
${ }^{17}$ Supra note de bas de page 7.

${ }^{18}$ Ibid.

La Benefit Corporation permet aux actionnaires d'engager les poursuites judiciaires contre ses dirigeants s'ils n'ont pas poursuivi le bénéfice public général ou des bénéfices publics spécifiques.
} 
permis de relativiser leur utilité (2.1). Cette forme juridique n'existant pas en France il est pourtant intéressant de se demander si leur introduction pourrait permettre une meilleure prise en compte par les entreprises de leur impact social et environnemental (2.2)?

\subsection{Une forme juridique à l'utilité controversée}

Le caractère novateur de la Benefit corporation n'est pas lié au fait que cette forme juridique impose aux créateurs qui se rattachent à ce statut de concilier finalités économiques et finalités environnementales et sociales. Il est lié à la nécessité d'avoir recours à une organisation tiers pour certifier son utilité environnementale ou sociale. Le gouvernement n'a pas pour rôle de vérifier le standard tiers choisi ou de superviser l'engagement responsable de la Benefit corporation. Il est cependant nécessaire de souligner les limites et les obstacles à l'articulation de cette double mission de la Benefit Corporation. D'abord, l'objectif de générer un «bénéfice public général » est défini d'une manière générale, donc peu précise (Cummings, 2012, p. 606; Munch, 2012, p. 171). La question de savoir si une Benefit Corporation poursuit sa vocation dépend d'une tierce partie non réglementée qu'elle choisit elle-même. La loi prévoit uniquement la référence à une tierce partie indépendante et transparente ${ }^{19}$.

L'indépendance implique entre autres l'absence de relations matérielles directes ou indirectes avec la Benefit corporation ou ses filiales. Un tiers indépendant n'est pas 1) un employé ou le directeur de la Benefit corporation ou d'une ses filiales, 2) lié à une relation familiale avec les dirigeants, 3 ) un actionnaire avec $5 \%$ ou plus des actions.

La transparence implique que le tiers indépendant rende publiques quatre types d'informations sur 1) les critères pris en compte lors de la mesure de la performance sociale et environnementale de la Benefit corporation, 2) la pondération relative de ces critères, 3) l'identification des personnes qui conduisent la révision du standard et, 4) le processus de sa révision.

Il est donc possible de supposer qu'une tierce partie pourrait élaborer des critères souples voire «mou» permettant à des entreprises de bénéficier de l'enveloppe de la Benefit Corporation tout en n'ayant qu'une performance sociétale faible. La souplesse présente l'avantage de permettre à chaque entreprise d'adapter la notion de

\footnotetext{
${ }^{19}$ Model Benefit Corporation Legislation
} 
«bénéfice public général » en fonction de ses particularités au risque de faire perdre toute valeur au statut des Benefit Corporation.

Par ailleurs, le rôle de l'organisme tiers indépendant s'agissant du contrôle de la mise en œuvre du "bénéfice public spécifique » n'est pas clair. En effet, les modalités de leur intervention ne sont pas précisées par les règlementations des États. Cet organisme tiers indépendant peut se contenter d'intervenir uniquement lors de la création de la Benefit corporation ou au contraire accompagner celleci lors de son activité en évaluant la performance sociétale.

Une autre limite du modèle hybride proposé par la Benefit Corporation tient à la nécessité de trouver un équilibre entre la recherche du profit et les objectifs sociétaux ou environnementaux (André, 2012, p. 138; Munch, 2012, p. 189). La loi actuelle donne peu d'indications, que ce soit aux actionnaires ou aux dirigeants, sur la manière dont les deux missions doivent être équilibrées. Cela pourrait permettre aux dirigeants de renoncer à la maximisation du profit en faveur de la performance sociale ou vice-versa. Compte tenu du fait que les dirigeants ont l'obligation de considérer les intérêts des différentes parties prenantes, nécessairement hétérogènes et parfois contradictoires, ils peuvent éviter de prendre certaines décisions en accordant la priorité à certaines parties prenantes. Cette situation peut leur donner un pouvoir discrétionnaire important. Ce pouvoir sera contrebalancé par celui des actionnaires qui choisissent délibérément cette forme d'entreprise. Ils disposent de pouvoirs importants car ils ont le droit de demander un renforcement des principes de gouvernance «Benefit Corporation», et de mener une action en justice en cas d'échec, de non-respect des objectifs ou de manque de transparence. Néanmoins, le contrôle par les actionnaires n'est pas une garantie contre un détournement de cette forme juridique. Une Benefit Corporation qui commencerait à s'écarter de sa double mission afin de privilégier les intérêts financiers pourrait augmenter les profits financiers ce qui est traditionnellement le but recherché dans une gouvernance actionnariale. Il n'est donc pas évident que ceux-ci envisagent les possibilités de contrôle dont ils disposent pour cadrer l'action des dirigeants. La recherche d'un profit sans règle rendrait difficile la compatibilité entre la logique financière de l'entreprise avec la logique sociale et environnementale (Boutinet et Bréchet, 2012, p. 19). Elle pourrait même faire prendre des risques aux profits financiers à long terme en négligeant la logique sociale et environnementale (risque pour l'image, de condamnation judiciaire...). Le profit doit être lié à une responsabilité envers la collectivité (Perroux, 1926). La multiplication des standards, non nécessairement convergents pose d'autres types de problèmes 
s'agissant du fonctionnement de la Benefit Corporation : celui du reporting et du contrôle des résultats sociaux et environnementaux (Cummings, 2012, p. 600). Les dirigeants peuvent tenter d'occulter la réalité par la présentation de fausses informations dans leurs « rapport annuel des bénéfices ».

Pour s'assurer que l'entreprise ne dissimule des engagements non réalisés, la loi régissant la Benefit Corporation devrait indiquer expressément les sanctions à l'encontre de toute personne qui diffuserait au public des informations fausses et trompeuses. Une vérification du rapport pourrait par ailleurs être pratiquée par un audit social indépendant (Brown et al., 2001, p. 291). La présence d'administrateurs indépendants, au sein de la Benefit Corporation, permettrait aussi d'examiner si l'information préparée est transparente et fidèle à la réalité. Les législations de l'État du Vermont et celle d'Hawaii énoncent déjà ce principe en exigeant un administrateur indépendant, désigné «benefit director ${ }^{20}$. D'autres États devraient également envisager d'adopter un tel dispositif.

Enfin, la Benefit Corporation peut agir comme une marque distinctive en offrant aux consommateurs, partenaires et employés un moyen facile d'identifier une vraie entreprise responsable. De ce fait, la Benefit Corporation ne peut être et rester un élément de valorisation pour une entreprise que si des règles d'entrées et des conditions sont établies pour sauvegarder la valeur du concept. L'absence de réglementation ainsi qu'une liberté d'utilisation non contrôlée de cette nouvelle forme d'entreprise risquerait de conduire à son emploi injustifié et donc à une dépréciation de sa valeur. En effet, tout symbole de qualité, qui n'est pas régit par des règles et encadré par des normes, va attirer des entreprises voulant s'attribuer l'effet marketing que va avoir ce modèle sur les consommateurs de plus en plus soucieux de la dimension environnementale et sociale. Il y a donc un lien entre la pérennité et l'efficacité d'une organisation telle que la Benefit Corporation et les exigences applicables aux entreprises qui vont se rattacher à ce statut juridique. De façon générale plus l'accès

\footnotetext{
20 "A benefit director shall be independent of and shall have no material relationship with the sustainable business corporation", A bill for an Act, state of Hawaii § 7(d).

"A benefit director shall be elected and may be removed in the manner provided by subchapter 1 of chapter 8 of this title and shall be an individual who is independent of the benefit corporation", An act relating to the Vermont Benefit Corporations Act. $\S$ 21.10 .

Voir site de la Benefit Corporation, http://www.benefitcorp.net/state-by-statelegislative-status
} 
va être restrictif, plus son effet de qualité va être important et pertinent. C'est par ce point-là que la Benefit Corporation va atteindre son but de qualité et va être reconnue au niveau des consommateurs et des entreprises.

Cette nouvelle forme juridique n'existe pas en France. Il est dès lors légitime de se demander si son intégration en droit français est utile afin d'encourager les entreprises à s'engager sur le plan social et environnemental.

\subsection{Vers une meilleure prise en compte en France de l'impact social et environnemental des entreprises par l'introduction de la Benefit Corporation?}

Les entreprises communiquent de plus en plus sur des finalités sociales ou environnementales ou en tout cas sur une prise en considération de ces questions. Elles ont donc tout intérêt à développer une démarche permettant d'apprécier l'adéquation de leurs comportements aux problématiques sociales et environnementales. La règlementation française ne leur fournit pas pour le moment d'outil adapté à cette intégration.

En droit français la définition de l'entreprise se confond avec celle de la société (article 1832 du Code civil). Cette considération est inexacte ou du moins imprécise. L'entreprise est en effet une notion bien connue des économistes et des gestionnaires mais elle n'a en tant que telle pas d'existence en droit (Robé, 2011, p. 19 et s.). L'entreprise peut exister au travers de plusieurs supports juridiques (sociétés civiles ou commerciales, personnes physiques ayant une activité économie...) et l'organisation humaine que constitue une entreprise ne se résume pas aux supports juridiques qui lui permettent d'exercer son activité. Ces questions expliquent la réflexion engagée pour donner un statut juridique à l'entreprise réelle (Roger, 2012, p. 23). Dans ces propos, nous circonscrirons la notion d'entreprise à celle de société commerciale, la société étant une réalité juridique «parallèle » à la notion d'entreprise (Brechet et Tougeron, 2014). L'entreprise accède à la vie juridique grâce au contrat de société. Ce contrat est conclu pour affecter à une entreprise commune des biens ou leur industrie en vue de partager le bénéfice ou de profiter de l'économie qui pourra en résulter. Nous sommes donc face à des personnes qui organisent leur coopération en mutualisant leurs ressources et en associant leurs compétences.

La recherche du lucre ou, pour être plus exact, la recherche d'une répartition des bénéfices est un élément fondamental en droit 
des sociétés français. La définition légale du contrat de société ne fait pas mention d'une quelconque recherche d'un «bénéfice public général ».

Pourtant en France des entreprises sont à la recherche d'un «bénéfice public général». Il en est ainsi des entreprises de l'économie sociale et solidaire (ESS). Ces entreprises ont en effet pour particularité le fait que la recherche du profit n'est pas l'objectif essentiel de leur action (Autès, 2006, p. 94). Jusqu'à la fin du XIXème siècle, les organisations qui n'avaient pas d'objectif lucratif devaient «s'ajuster au modèle de la société, ou n'exister que de fait » (Castro, 1996, p. 53), progressivement le législateur a ensuite institué des organisations à but non-lucratif (associations, fondations...). Il est toutefois difficile d'identifier juridiquement les entreprises de l'ESS (Branellec, 2013, p. 104). En effet, il n'y a pas un mais de multiples supports juridiques possibles pour une entreprise de l'ESS. Il est possible d'y trouver aussi bien des formes civiles (association, mutuelle...) que commerciales (société à responsabilité limitée, société anonyme, société coopérative d'intérêt collectif...), à tel point que l'ESS est parfois qualifiée de «secteur multiforme » ${ }^{21}$. La création d'un statut unique pour l'ensemble des entreprises de l'ESS a d'ailleurs été imaginée mais écartée. D'une part, le secteur de l'ESS ne semble pas en faire la demande, ce qui montre bien que ses acteurs trouvent des outils juridiques ad hoc au sein du corpus juridique (Lafore, 2003). D'autre part, il semble impossible d'arriver à mettre en place un modèle juridique uniforme qui pourrait servir de support à la fois à des ateliers d'insertion, des clubs sportifs, des régies de quartiers, des systèmes d'échange locaux ou un groupement de producteurs agricoles.

Toutefois, dans tous les cas, les entreprises de l'ESS vont rechercher l'efficacité de leur action. Lorsqu'elles s'inscrivent dans une logique de marché, elles rechercheront la viabilité économique de leur activité. Lorsqu'elles visent la réalisation d'un profit, celui-ci va être un moyen d'accomplir une action. L'action de ces entreprises va tendre à l'utilité sociale. L'entrepreneur social est ainsi défini par la School for Social Entrepreneurs comme « celui qui opère de manière entrepreneuriale mais pour le bien public ou d'une collectivité plutôt que pour s'enrichir ».

L'utilité sociale est « une notion dérivée de celle de l'intérêt général » (Euillet, 2002, p. 210). Elle ne doit pas être confondue avec la notion d'intérêt social. L'intérêt social est «entendu comme

\footnotetext{
${ }^{21}$ Rapport de la mission parlementaire sur le développement de l'économie sociale et solidaire, présidée par Francis Vercamer, remis au gouvernement le 28 avril 2010.
} 
l'intérêt supérieur de l'entreprise dépassant les intérêts catégoriels de tous ses membres » (Daigre 1996, p. 24). C'est l'équivalent de la notion de «going concern» développée par la jurisprudence américaine selon laquelle chacun a intérêt à contribuer aux buts de l'entreprise au «bien commun» car la satisfaction de ses propres intérêts en dépend. Dans la conception institutionnelle de la société, les intérêts individuels sont subordonnés à l'intérêt social (Champaud, 1991, p. 48) puisque la poursuite de cet intérêt supérieur conditionne la satisfaction des intérêts spécifiques (Desreumaux et Brechet, 2013, p. 83 et 84 ). Cette notion est utile puisqu'elle peut servir au juge de boussole pour trancher entre des intérêts particuliers (en cas notamment de conflits entre des associés minoritaires et majoritaires dans le cadre d'une action judiciaire en abus de minorité ou en abus de majorité).

La notion d'intérêt général est différente, elle illustre la prise en compte actuelle de l'extérieur par les entreprises (Hiez et Laurent, 2011, p. 36). La notion d'intérêt général, «épine dorsale du droit public » (Walline, 1999, p. 277), va progressivement céder le pas à celle d'utilité sociale créée au profit de catégories mouvantes et mal définies d'usagers (Parodi, 2010, p. 43) et qui va reposer sur un choix de valeur. Il en résulte que cette notion d'utilité sociale n'est pas une notion juridique; elle est malaisée à définir car protéiforme et contingente. L'utilité sociale peut se manifester ou se cristalliser à quatre niveaux: un premier dans lequel elle rejoindra l'utilité individuelle, un second dans lequel elle sera une utilité collective, à un troisième niveau l'utilité sera sociale externe et à un dernier niveau l'utilité sera sociétale (halo sociétal) (Parodi, 2010, p. 44-46). Ces différents niveaux montrent bien que l'utilité sociale ne saurait être réduite à l'utilité individuelle ou collective mais qu'elle « déborde de toute part dans les cercles concentriques du halo sociétal ou les composantes de la société » (Parodi, 2010, p. 54). Cette ouverture sur l'extérieur rejoint l'idée que l'entreprise solidaire et sociale a une responsabilité et une finalité sociales. Les entreprises de l'ESS ont ainsi un devoir envers la collectivité à laquelle elles empruntent des ressources pour leur financement. Cette démarche responsable rejoint la montée en puissance du concept de responsabilité sociale de l'entreprise : celle-ci devant être « citoyenne».

La prise en compte par les entreprises capitalistes des questions de RSE et la multiplication des démarches RSE initiées font craindre que celles-ci ne soient que des artéfacts publicitaires, "que le quantitatif ou le marketing prime sur la qualité »(Delga, 2005, p. 8). Il y a dès lors un risque de perte de lisibilité entre les entreprises de l'ESS et celles capitalistes. 
Les enjeux financiers et politiques doivent donc pousser les entreprises à mesurer et à pouvoir justifier de leur utilité sociale. Le principal problème qui se pose est que l'utilité sociale est une notion extra-financière et donc difficilement quantifiable. Pourtant, la lisibilité de cette utilité est le gage d'une bonne comparabilité et d'une meilleure gouvernance des entreprises.

Cette démarche d'objectivisation de l'utilité sociale et environnementale a été entreprise dès les années 2000 par le législateur français. Ainsi la loi $\mathrm{n}^{\circ}$ 2001-420 du 15 mai 2001 relative aux nouvelles régulations économiques (loi NRE) est une consécration légale des démarches volontaires de diffusion d'informations initiées par les entreprises. La loi NRE a d'abord imposé une transparence environnementale aux entreprises cotées sur un marché réglementé en leur enjoignant de faire figurer dans un rapport des informations environnementales et sociales. La loi Grenelle 2 et le décret $n^{\circ} 2012$ 557 du 24 avril 2012 relatif aux obligations de transparence des entreprises en matière sociale et environnementale ont ensuite étendu cette obligation aux SA et SCA qui dépassent des seuils fixés à 100 millions d'euros pour le total du bilan, 100 millions d'euros pour le montant net du chiffre d'affaires et à 500 pour le nombre moyen de salariés permanents employés au cours de l'exercice (la mise en œuvre de ce dispositif est différenciée et échelonnée).

Il s'agit ici de l'application d'un principe « rendre compte pour mieux prendre en compte»(Blin-Franchomme, 2008, $\mathrm{n}^{\circ}$ 32). Le rapport de gestion doit exposer les actions menées et les orientations prises par la société afin qu'elle remplisse ses engagements sociétaux en faveur du développement durable. Les entreprises doivent donner de l'information sur des thèmes dont la liste est prévue par le décret du 24 avril 2012. Une première liste concerne toutes les sociétés assujetties à l'obligation de reporting et une seconde liste uniquement les sociétés dont les titres sont admis aux négociations sur un marché règlementé. Une société peut tout à fait ne pas renseigner certains thèmes à condition dans ce cas de se justifier. Il s'agit d'une transposition du principe anglo-saxon comply or explain. Ce dispositif a pour effet de permettre aux parties prenantes de prendre connaissance des informations sociales et environnementales qui concernent l'activité des sociétés. Elle doit permettre également de comparer de manière objective les performances extra-financières des entreprises.

Cette loi reste toutefois une lex imperfecta (Cuzacq, 2013, p. 15) car les parties prenantes peuvent tout à fait ne pas intégrer ces informations dans leurs arbitrages. Par ailleurs les informations peuvent très bien n'être que lacunaires car les entreprises peuvent ne 
pas renseigner certains thèmes, elles ont également la possibilité de définir elles-mêmes le contenu des indicateurs. La Benefit Corporation permettrait de corriger ces imperfections puisqu'elle impose aux entreprises d'inscrire une mission sociale et/ou environnementale dans ses statuts. Les dirigeants pourraient donc voir leur responsabilité mise en jeu s'ils se contentent d'utiliser les ressources de l'entreprise pour maximiser les profits dévolus aux actionnaires.

\section{Conclusion}

En définitive, il apparaît fondé que la France, et de façon plus large l'Union européenne, engagent un débat sur la question de la création d'un statut juridique adapté à des sociétés capitalistes ayant une utilité sociale et environnementale.

L'intérêt de cette forme juridique est qu'elle est adoptée de façon volontaire par les actionnaires et les dirigeants et qu'elle leur laisse le soin de déterminer les objectifs sociaux et environnementaux qu'ils se fixent. Ce droit laissant aux acteurs économiques le soin de s'engager volontairement et de décider quelle prendra la forme de leur engagement semble adapté à une économie libérale dans laquelle les entreprises doivent maintenir leur compétitivité. Ce choix est d'ailleurs plébiscité par l'OCDE et l'Union européenne (MaljeanDubois, 2008, p. 37).

La France a d'ailleurs pour le moment fait le choix de ne pas imposer de règlementation contraignante en matière de RSE en imposant uniquement aux entreprises une transparence sur leurs actions et leurs impacts par le biais du reporting extra-financier. La Benefit Corporation irait dans le sens de cette transparence en permettant à des entreprises de bénéficier d'une forme juridique qui garantisse qu'elles concilient la performance financière avec celle environnementale et sociale.

Cette consécration de la volonté des acteurs serait une nouvelle marque de la diminution de la hard law en droit des sociétés et conduirait «à confirmer les transformations pressenties d'un droit devenu post-moderne, d'une puissance publique relayée par la gouvernance, d'une unilatéralité marquant le pas face à la négociation, d'outils juridiques cédant la place à des outils économiques » (Belrhali-Bernard, 2009, p.1683).

\section{Bibliographie}


Aglietta M. et REBERIOUX A., Dérives du capitalisme financier, Editions Albin Michel, Paris, 2004.

American Law Institute, Principles of Corporate Governance: Analysis and recommendations, American Law Institute Publishers, St. Paul, MN, 1994.

ANDRÉ R., «Assessing the Accountability of the Benefit Corporation: Will This New Gray Sector Organization Enhance Corporate Social Responsibility? », Journal of Business Ethics, Vol. 110, Issue1, 2012, p133.-150.

AUTES M., «Les acteurs et les référentiels », in CHOPART J. N., NEYRET G. et RAULT D., (dir.), Les dynamiques de l'économie sociale et solidaire, La Découverte, Paris, 2006, p81.-113.

BLAIR M. M., Ownership and control: Rethinking Corporate Governance for the Twenty-First Century, Brookings Institution Press, Washington D.C, 1995.

BELRHALI-BERNARD H., «Le droit de l'environnement: entre incitation et contrainte », $R D P, \mathrm{~N}^{\circ} 6,2009$, p1683.-1704.

BLIN-FRANCHOMME M. P., «Entreprise et responsabilité : aperçu de quelques avancées récentes du développement durable dans la vie des affaires », Revue Lamy droit des affaires, №32, 2008, p61.-67.

BLIN-FRANCHOMME M. P., «De quelques éléments de régulation des démarches volontaires en matière de RSE... », Revue Lamy droit des affaires, $\mathrm{N}^{\circ} 40,2009$, p71.-74.

BOUTINET J. P. et BRECHET J. P., «Logiques de projet et logiques de profit : compatibilité et incompatibilité », hal-00732929, 2012.

BRANELLEC G., "Vers une justification par les entreprises de leur appartenance au secteur de l'économie sociale et solidaire », Gestion 2000, Vol.30, N², 2013, p103.-119.

BReCheT J. P. et TOUgERON P. Y., «Pouvoir managérial et gouvernance dans l'entreprise patrimoniale cotée », hal-00421220, 2009.

Brechet J. P. et Tougeron P. Y., «De quoi les parties sont-elles prenantes? ", Colloque de l'Association Internationale de Management Stratégique (AIMS), Rennes, 26-28 mai 2014.

BROWN A., KOK P., MCKENNA R. et VAN DER WIELE T., « A corporate social responsibility audit within a quality management framework », Journal of Business Ethics, Vol.31, ํㅜㄴ, 2001, p285.-297.

California Corporate Flexibility Act of 2011.

CASTRO S., "Le tiers secteur face au droit», in ARCHAMBAUlt E., (dir.), Le secteur sans but lucratif, Economica, Paris, 1996.

CHAMPAUD C., "L'intérêt social, principe général du droit des sociétés », RTD Com. 1991, p.58. 
CHARREAUX G., «Vers une théorie du gouvernement des entreprises ", in CHARREAUX G., (dir.), Le gouvernement des entreprises - Corporate Governance : théorie et faits, Economica, Paris, 1997, p421.-469.

CHARREAUX G. et DESBRIERES P., "Gouvernance des entreprises : valeur partenariale contre valeur actionnariale », Finance Contrôle Stratégie $1, \mathrm{~N}^{\circ} 2,1998$, p57.- 88.

CLARK W. H., Canonchet Group LLC, Drinker Biddle \& Reath LLP et VRANKA L., "White Paper, The Need and Rationale for the Benefit Corporation: Why it's the Legal Form that Best Addresses the Needs of Social Entrepreneurs, Investors, and, Ultimately, the Public », 2013 CumMings B., «Benefit Corporations: How to Enforce a Mandate to Promote the Public Interest », Columbia Law Review, Vol.112, 2012, p578.-627.

CUZACQ N., «Les nouveautés issues du décret $n^{\circ} 2012-557$ du 24 avril 2012 relatif aux obligations de transparence des entreprises en matière sociale et environnementale », Revue de droit du travail, $\mathrm{N}^{\circ} 1$, 2013, p15.-25.

DAIGRE J. J., «Le gouvernement d'entreprise : feu de paille ou mouvement de fond ? », Droit et patrimoine, juillet-août 1996, p24.

DAY J. et SINGER D. E., «New York Benefit Corporations: Bringing social responsibility to business », The CPA Journal, mars, 2014, p4043.

DELGA J., «Propos iconoclastes sur l'éthique d'entreprise: Une analyse juridique de l'éthique d'entreprise au regard de la mondialisation », Revue Lamy Droit des affaires, N84, 2005, p5.-9.

DESBARATS I., «Regard sur un instrument majeur de la gouvernance d'entreprise : quid de la nature juridique des codes éthiques ? ", Revue Lamy droit des affaires, $\mathrm{N}^{\circ} 32$, 2008, p68.-70.

DESREUMAUX A. et BRECHET J.-P., «L'entreprise comme bien commun », RIMHE, 2013, n 7, p. 77-93.

ESPOSITO R. T., «The Social Enterprise Revolution in Corporate Law: A Primer on Hybrid Corporate Entities in Europe and the United States and the Case for the Benefit Corporation », William \& Mary Business Law Review, Vol.4, 2013, p639.-714.

ESPOSITO R. T. et PELSINGER S., "The Supreme Court's first brush with social enterprise », Stanford social innovation review, $\mathrm{N}^{\circ} 6,21$ juillet 2014.

EUILLET A., "L'utilité sociale, une notion dérivée de celle d'intérêt général », Revue de droit sanitaire et social, Vol.38, N², 2002, p207.-228. 
HAFSI T. et YoussofZAI F., «Dirigeants d'entreprises, focaliser sur les actionnaires n'est pas légitime! », Revue française de gestion, $\mathrm{N}^{\circ} 183,2008, \mathrm{p} 111 .-130$.

HIEZ D. et LAURENT R., « La nouvelle frontière de l'économie sociale et solidaire : l'intérêt général ? », Recma, N³19, 2011, p36.-56.

LAFORE R., «La mise en forme juridique », in ITÇAINA X., LAFORE R. et SORBETS C., (dir.), Générer et gérer du bien collectif en Aquitaine. Un monde en quête de reconnaissance, CERVL, IEP de Bordeaux, 2003, p.45.

LEVILLAIN K., « La Flexible purpose corporation. Un petit pas pour le juriste, un grand pas pour l'entreprise ? », Cadres-CFDT, $\mathrm{N}^{\circ} 450-451$, 2012, p15.-24.

MALJEAN-DUBOIS S., Quel droit pour l'environnement, Hachette, Paris, 2008.

MARTINET A. C., «Gouvernance et management stratégique. Une nouvelle science morale et politique », Revue française de gestion, Vol.3, N 183,2008 , p95.-110.

Model Benefit Corporation Legislation, 2012.

MUNCH S., «Improving the Benefit Corporation: How Traditional Governance Mechanisms Can Enhance the Innovative New Business Form », Northwestern Journal of Law \& Social Policy, Vol.7, Issue1, p170.-195.

PARODI M., «L'utilité sociale pour éclairer la face cachée de la valeur de l'économie sociale », Recma, №315, 2010, p40.-55.

Pennsylvania Bar Association, House Bill 1616, Corporations and Unincorporated Associations of the Pennsylvania Consolidated Statutes, further providing for benefit corporations, 2010.

PEREIRA B., "Chartes et codes de conduite : le paradoxe éthique », Revue Management et avenir, $\mathrm{N}^{\circ} 21,2009, \mathrm{p} 26 .-43$.

PERROUX F., Le problème du profit, PUG, Paris, 1926 (Nouv. éd).

RESOR F. R., «Comment. Benefit corporation legislation », Wyoming Law Review, Vol.12, p91.-113.

ROBE J. P., « L'entreprise oubliée par le droit », Le Journal de l'Ecole de Paris, $\mathrm{N}^{\circ} 32$, nov. déc. 2001, p19.-37.

ROGER B. (éd., Collège des Bernardins), L'entreprise, formes de la propriété et responsabilités sociales, Editions Lethielleux, Paris, 2012.

SCHMIDT E., «Vermont's Social Hybrid Pioneers: Early Observations and Questions to Ponder », Vermont Law Review, Vol.35, No.1, 2010, p63.-209.

State of Maryland, Maryland General Assembly, House Bill 1009, Corporations-Benefit Corporation, 2010. 
TIROLE J., «Corporate Governance », Econometrica, Vol.69, No.1, 2001, p1.-35.

WALINE M., «L'intérêt général », rapport public du Conseil d'Etat, 1999.

ZINGALES L., «Corporate governance », Working Paper 6309, National Bureau of Economic Research, Cambridge, MA, 1997. 